\title{
Systematic re-analysis strategy of serum indices identifies alkaline phosphatase as a potential predictive factor for cervical cancer
}

\author{
JUN YU $^{1 *}$, QIANWEN ZHENG $^{1^{*}}$, XIAOXING DING $^{1^{*}}$, BO ZHENG $^{2 *}$, XIA CHEN $^{1 *}$, BINGHAI CHEN $^{3 *}$, \\ CONG SHEN $^{2}$, YU ZHANG ${ }^{1}$, XIAOJIN LUAN ${ }^{1}$, YIDAN YAN ${ }^{1}$, WANYIN CHEN ${ }^{1}$, BING XIE $^{1}$, MIN WANG $^{1}$,

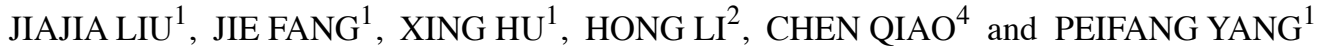 \\ ${ }^{1}$ Department of Gynecology, Affiliated Hospital of Jiangsu University, Zhenjiang, Jiangsu 212001; \\ ${ }^{2}$ Center for Reproduction and Genetics, Suzhou Municipal Hospital, Nanjing Medical University Affiliated \\ Suzhou Hospital, Suzhou, Jiangsu 215002; Departments of ${ }^{3}$ Urology and ${ }^{4}$ Clinical Pharmacy, Affiliated \\ Hospital of Jiangsu University, Zhenjiang, Jiangsu 212001, P.R. China
}

Received February 20, 2018; Accepted June 4, 2019

DOI: $10.3892 / \mathrm{ol} .2019 .10527$

\begin{abstract}
The aim of the present study was to identify predictive factors for cervical cancer (CC) progression using a multistage approach. The present study obtained data from 390 healthy women and 259 patients with cervical cancer between June 2012 and June 2017, and used a multiple stage re-analysis strategy for clinical detection of CC. A total of seven types of serum indices were used in the present study, including sugar chain antigen 125 (CA-125), sugar chain antigen 199 (CA-199), $\alpha$ fetoprotein (AFP), carcinoembryonic antigen, alkaline phosphatase (ALP), cholesterol and triglyceride (TG). The expression levels of CA-125, CA-199, AFP, ALP, cholesterol and TG were significantly different between healthy women and patients with cervical squamous cell carcinoma (SCC). Furthermore, ALP, cholesterol and TG expression levels were significantly different
\end{abstract}

Correspondence to: Dr Jun Yu or Professor Peifang Yang, Department of Gynecology, Affiliated Hospital of Jiangsu University, Jiangsu University, 438 Jiefang Road, Zhenjiang, Jiangsu 212001, P.R. China

E-mail: yujun9117@126.com

E-mail: ypf@ujs.edu.cn

${ }^{*}$ Contributed equally

Abbreviations: CC, cervical cancer; FIGO, The International Federation of Gynecology and Obstetrics; SCC, squamous cell carcinoma; AC, cervical adenocarcinoma; ASC, adenosquamous cervical carcinoma; TG, triglyceride; CA-125, sugar chain antigen 125; CA-199, sugar chain antigen 199; AFP, $\alpha$ fetoprotein; CEA, carcino-embryonic antigen; SC, ectoendocervical squamocolumnar; AST, aspartate aminotransferase; SCCA, squamous cell carcinoma antigen; ALP, alkaline phosphatase; TNALP, tissue non-specific isozyme ALP

Key words: CC, SCC, AC, serum index, ALP, predictive factor in healthy women compared with patients with cervical adenocarcinoma (AC). Further comparisons based on age and pathological staging demonstrated that the variability in the ALP level was not significant between the $<40$ years old age group and the 40-50 years old age group within healthy individuals $(\mathrm{P}>0.05)$; however, was significant in patients with SCC $(\mathrm{P}<0.05)$. Staging analysis identified significant differences in ALP between healthy women and patients with SCC (Stage I-IV), and significant differences between healthy women and patients with Stage I AC. The results of the present study indicated that the expression of ALP was significantly increased in patients with CC compared with healthy women. Therefore, ALP may be a potential predictive factor for the development of CC.

\section{Introduction}

Cervical cancer (CC) is one of the most prevalent and malignant types of cancer in women, and is the second most common malignancy in women with $>50$ million new cases each year (1). Although cervical screening programs in certain developed countries have contributed to the detection and prompt treatment of cervical abnormalities, the morbidity and mortality of $\mathrm{CC}$ has continued to increase on a yearly basis, particularly in developing countries (2).

Tumor biomarkers have been used for the clinical diagnosis of cancer during the early stages, and have been demonstrated to greatly improve the effectiveness of treatments and thus the quality of life. Of these biomarkers, squamous cell carcinoma antigen (SCCA) is a predictive and prognostic factor for CC (3). However, SCCA exhibits low sensitivity, resulting in a relatively high risk of missed diagnosis of $\mathrm{CC}$ during screening $(4,5)$. A previous study indicated that the levels of SCCA were also increased in other types of cancer, including breast cancer (5). Sugar chain antigen 125 (CA-125) was used for the diagnosis of ovarian carcinoma and its sensitivity was primarily based on the staging of ovarian malignancies (6). However, it has been reported that the expression levels of CA-125 were also elevated in other malignancies, including 
$\sim 10.4 \%$ of benign ovarian tumors (7). Therefore, it is necessary to screen for more specific indicators that could predict the prognosis and assist in the diagnosis of $\mathrm{CC}$.

Researchers have attempted to identify more accurate and simple serum indices, such as serum vascular endothelial growth factor levels (8) and pre-treatment serum hemoglobin levels (9), to assist in the diagnosis and prognosis of CC. However, there remains a lack of specific predictors for the pathogenesis of $\mathrm{CC}$ and thus more useful markers are required for improved early diagnosis. Therefore, based on the results of the aforementioned previous studies $(8,9)$, the present study further investigated the predictive factors associated with $\mathrm{CC}$ progression.

The aim of the present study was to identify biomarkers to predict and detect progression of $\mathrm{CC}$ through re-analysis of clinical serum indices using a multistage approach. A total of four serological tumor markers, including sugar chain antigen 199 (CA-199), CA-125, $\alpha$-fetoprotein (AFP) and carcino-embryonic antigen (CEA) were selected in the present study to assess their diagnostic specificity for CC. Furthermore, the present study also analyzed three potential metabolic factors, including alkaline phosphatase (ALP), cholesterol and triglyceride (TG) for the prediction of $\mathrm{CC}$ progression. The present study systemically analyzed the association between seven types of serum indices and $\mathrm{CC}$, in order to assess whether they could be used as early prediction factors of $\mathrm{CC}$.

\section{Patients and methods}

Study population. The present study included 259 patients with CC and 390 healthy women recruited from June 2012 to June 2017 at The Affiliated Hospital of Jiangsu University (Zhenjiang, China). The characteristics of patients with CC and healthy control women are summarized in Table I. The pathological classification of $\mathrm{CC}$ was based on the morphology of cervical tissues and cells, and confirmed by two independent pathologists at The Affiliated Hospital of Jiangsu University. Patients with CC were divided into three groups: i) Stage I; ii) Stage II; and iii) Stage III/IV, according to the International Federation of Gynecology and Obstetrics (FIGO) staging system (10). Additionally, patients with CC and healthy controls were divided into three age groups: $<40$ years old, 40-50 years old and >50 years old. The present study was approved by The Ethics Committee for Biomedical Research at Affiliated Hospital of Jiangsu University (approval no. 20120019), and conducted according to the guidelines for clinical retrospective studies (11). All participants were fully informed of the purpose and significance of the study. Written informed consent was obtained from all the participants for participation.

Data collection. The accrual data collection was retrospective. Inclusion criteria for patients with $\mathrm{CC}$ were: i) Patients diagnosed with CC; ii) FIGO Stage I-IV; iii) data collection prior to radical surgery for $\mathrm{CC}$ from patients not treated with radiotherapy or chemotherapy; and iv) no abnormal fever or infection present. Exclusion criteria for patients with $\mathrm{CC}$ were: i) Data collection following radical surgery for $\mathrm{CC}$ or postoperative recurrent cases of $\mathrm{CC}$; ii) patients who have undergone radiotherapy or chemotherapy; iii) patients with other malignant tumors, including ovary masses and uterine fibroids; iv) patients with other diseases, including hepatitis, chronical disorder, inflammatory infection, endometriosis, uterine fibroid and cervical intraepithelial neoplasia, which would have affected serum indices used in the present study and v) patients exceeding the normal upper limit of aspartate aminotransferase (AST) and alanine transferase (ALT). The average age of patients with $\mathrm{CC}$ tested for serum indices was $50.6 \pm 11.3$ years. Data for healthy women were collected from the physical examination center, with an average age of $35.8 \pm 9.7$ years. No organic lesions or abnormal alterations were identified during the physical examination of the healthy women.

Hematoxylin and eosin $(H \& E)$ staining. Briefly, cervical tissues were fixed in $4 \%$ paraformaldehyde at room temperature for $40 \mathrm{~min}$, embedded in paraffin and cut into $5-\mu \mathrm{m}$-thick sections. After deparaffinization and rehydration, sections were stained with hematoxylin solution for $5 \mathrm{~min}$ followed by 5 dips in $1 \%$ acid ethanol $(1 \% \mathrm{HCl}$ in $70 \%$ ethanol) and then rinsed in distilled water at room temperature. Then the sections were stained with eosin solution for $3 \mathrm{~min}$ and followed by dehydration with graded alcohol and clearing in xylene at room temperature. The samples were mounted with neutral balsam and visualized using a bright-field fluorescent microscope (x100 magnification; Axioskop 2 Plus; Carl Zeiss AG, Oberkochen, Germany).

Detection of serum indices. For detection of serum indices, $24 \mathrm{~h}$ prior to commencement of the program, and $48 \mathrm{~h}$ after the last exercise training session and after $12 \mathrm{~h}$ of fasting, 5-ml blood samples were collected from the brachial veins of the participants between 8 and 10 a.m. Samples were poured into testing tubes without an anticoagulant, to allow the serum to separate. To minimize the time the samples were kept in laboratory conditions, after a 5-min incubation at room temperature, the sample was immediately centrifuged with $1,500 \mathrm{x} \mathrm{g}$ at $4^{\circ} \mathrm{C}$ for $5 \mathrm{~min}$ and the serum solution was divided after blood clotting. Subsequently, the resulting serum was used to determine the levels of CA-199, CA-125, AFP, CEA, ALP, cholesterol, TG, ALT, AST and estradiol. Serum indices were measured at the same time-point using an immunochemiluminescence detection system (i2000-SR; Abbott Pharmaceutical Co., Ltd., Lake Bluff, IL, USA) and an automated biochemical analyzer (AU5800; Beckman Coulter, Inc., Brea, CA, USA).

Reverse transcription-quantitative polymerase chain reaction $(R T-q P C R)$. Total RNA was isolated from cervical tissues using RNAiso Plus (Takara Biotechnology Co., Ltd., Dalian, China) according to the manufacturer's protocol. Total RNA was reverse transcribed into cDNA at $37^{\circ} \mathrm{C}$ for $15 \mathrm{~min}$ followed by $85^{\circ} \mathrm{C}$ for $5 \mathrm{sec}$ using a Superscript RT kit (Takara Biotechnology Co., Ltd.) and amplified using the SYBR Premix Ex Tag ${ }^{\text {TM }}$ kit (cat. no. RR420B; Takara Biotechnology Co., Ltd.), under the following conditions: Pre-denaturation at $95^{\circ} \mathrm{C}$ for $30 \mathrm{sec} ; 40$ cycles of denaturation at $95^{\circ} \mathrm{C}$ for $3 \mathrm{sec}$, annealing at $60^{\circ} \mathrm{C}$ for $30 \mathrm{sec}$ and extension at $72^{\circ} \mathrm{C}$ for $60 \mathrm{sec}$. The following primers were used for the amplification of tissue 
Table I. Characteristics of normal controls and patients with CC.

\begin{tabular}{|c|c|c|c|c|}
\hline \multirow[b]{2}{*}{ Characteristic } & \multirow[b]{2}{*}{ Normal } & \multicolumn{3}{|c|}{$\mathrm{CC}$} \\
\hline & & SCC & $\mathrm{AC}$ & ASC \\
\hline Participants, n (\%) & 390 & $235(90.7 \%)$ & $23(8.9 \%)$ & $1(0.4 \%)$ \\
\hline Age, mean \pm standard deviation & $35.8 \pm 9.7$ & $50.7 \pm 11.3$ & $50.8 \pm 11.0$ & 38 \\
\hline \multicolumn{5}{|l|}{ Age, $n$} \\
\hline$<40$ & 258 & 32 & 5 & 1 \\
\hline $40-50$ & 94 & 102 & 7 & N/A \\
\hline$>50$ & 38 & 101 & 11 & N/A \\
\hline FIGO stage, n (\%) & N/A & $235(90.7 \%)$ & $23(8.9 \%)$ & $1(0.4 \%)$ \\
\hline I & N/A & $119(45.9 \%)$ & $16(6.2 \%)$ & $\mathrm{N} / \mathrm{A}$ \\
\hline II & N/A & $90(34.7 \%)$ & $6(2.3 \%)$ & N/A \\
\hline III/IV & N/A & $26(10.1 \%)$ & $1(0.4 \%)$ & $1(0.4 \%)$ \\
\hline
\end{tabular}

CC, cervical cancer; SCC, squamous cell carcinoma; AC, cervical adenocarcinoma; ASC, adenosquamous cervical carcinoma; FIGO, The International Federation of Gynecology and Obstetrics.

non-specific isozyme ALP (TNALP): Forward, 5'-ACTCTC CGAGATGGTGGTGGTG-3' and reverse, 5'-CGTGGTCAA TTCTGCCTCCTTCC-3'. GAPDH was used as an internal control with the following primers: Forward, 5'-ACCCAG AAGACTGTGGATGG-3' and reverse, 5'-TTCAGCTCAGGG ATGACCTT-3'. The relative changes in gene expression were calculated using the $2^{-\Delta \Delta C q}$ method (12).

Statistical analysis. Data are presented as the mean \pm standard deviation. Odds ratios (ORs) and 95\% confidence intervals (CIs) were analyzed using logistic regression. Differences between two groups were calculated using a Student's t-test. Differences for the comparison of multiple groups were calculated using one-way ANOVA with a post-hoc Bonferroni test. Data were analyzed using SPSS software (version 22; IBM Corp., Armonk, NY, USA). Experiments were repeated at least three times. $\mathrm{P}<0.05$ was considered to indicate a statistically significant difference.

\section{Results}

Structure of cervical tissue in normal controls and patients with $C C$. To investigate the structure of cervical tissue in the normal controls and patients with $\mathrm{CC}$, cervical tissue was stained using H\&E. Histological analysis of the ectoendocervical squamocolumnar (SC) junction from normal adults revealed a discrete population of cuboidal epithelial cells at the interface of the ectocervix and endocervix (Fig. 1A). Previously, gene expression profiling separated the SC junction cells from the squamous and columnar cells, indicating significant differences between the two types of cells (13). In patients with cervical squamous cell carcinoma (SCC) and cervical adenocarcinoma (AC), the SC junction disappeared and was replaced with tumor cells (Fig. 1B and C).

Re-analysis of potential serum indices for $C C$. To identify novel clinical predictive factors for patients with $\mathrm{CC}$, the present study systemically used a multistage re-analysis
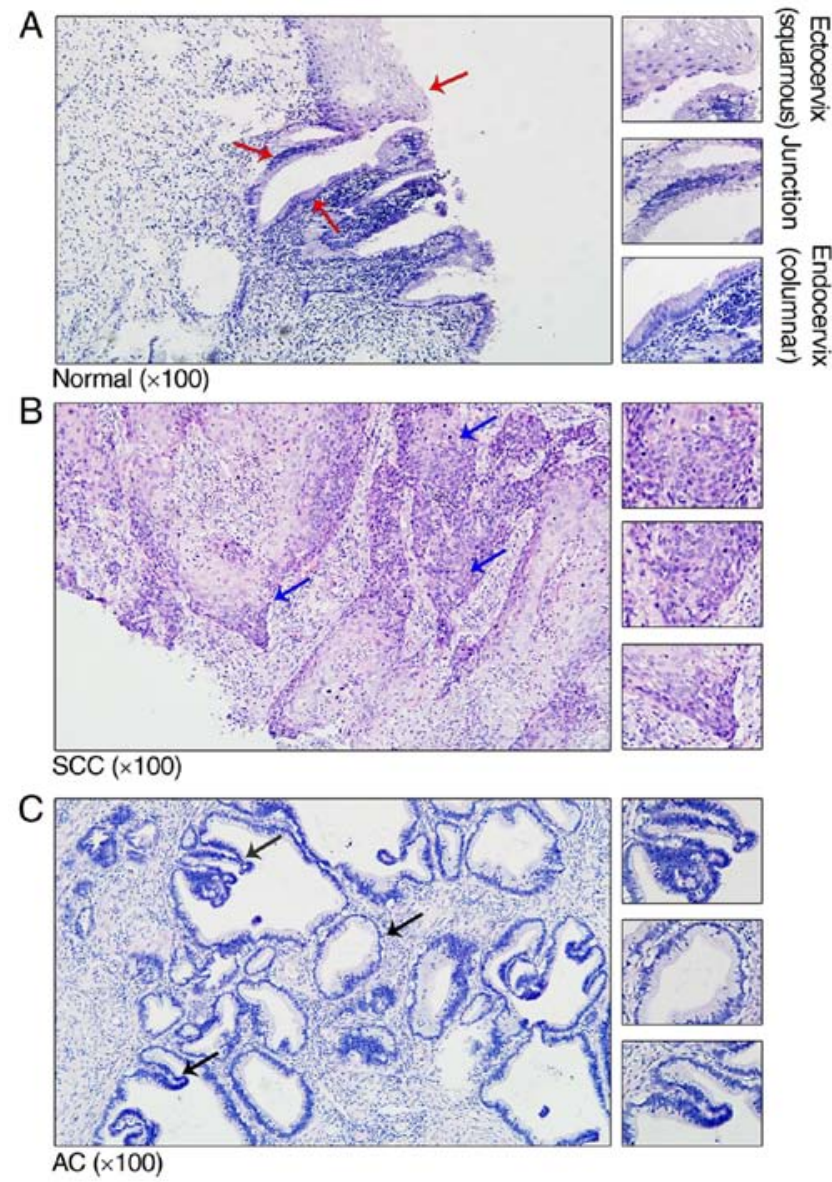

Figure 1. Representative histological haematoxylin and eosin staining of the adult cervix. (A) Normal cervical tissue with squamous, ectoendocervical squamocolumnar junction and columnar cells (red arrows). (B) SCC cervical tissue with representative nidus (blue arrows). (C) AC cervical tissue with representative nidus (black arrows). The magnified views (x200) are representative structures, which are indicated by the arrows. SCC, squamous cell carcinoma; AC, cervical adenocarcinoma.

strategy of seven serum indices (Fig. 2). A total of four serological tumor markers, including CA-199, CA-125, AFP 


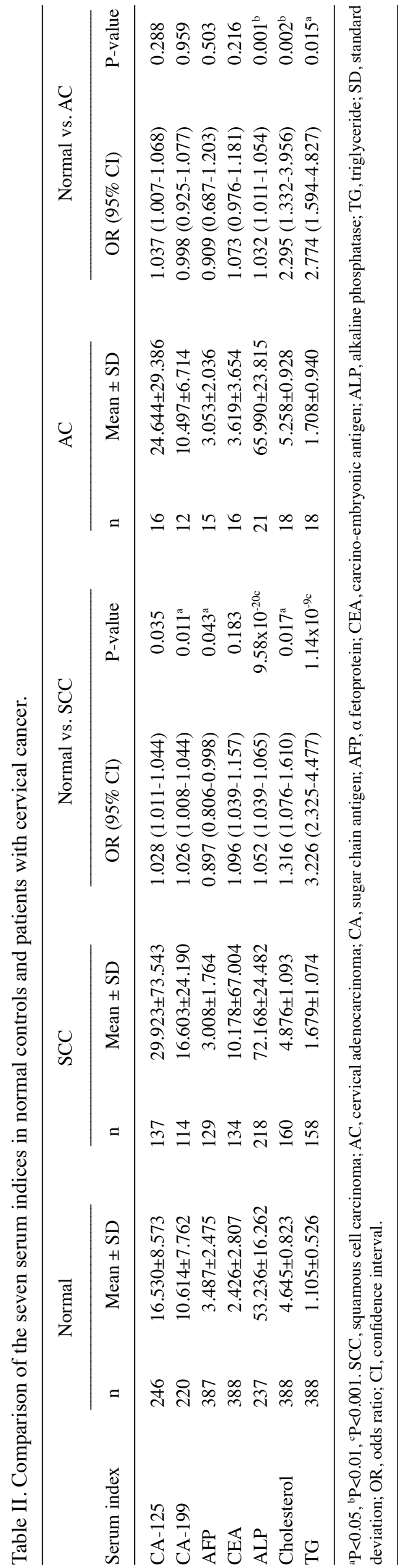

and CEA were used in the present study to assess their specificity for CC. Furthermore, three potential metabolic factors, including ALP, cholesterol and TG were also analyzed for the prediction of CC progression.

The expression levels of CA-125 $(\mathrm{P}=0.035), \mathrm{CA}-199$ $(\mathrm{P}=0.011)$, AFP $(\mathrm{P}=0.043)$, ALP $(\mathrm{P}<0.001)$, cholesterol $(\mathrm{P}=0.017)$ and $\mathrm{TG}(\mathrm{P}<0.001)$ were significantly different between healthy women and patients with SCC. Furthermore, for healthy women and patients with $\mathrm{AC}, \mathrm{ALP}(\mathrm{P}=0.001)$, cholesterol $(\mathrm{P}=0.002)$ and $\mathrm{TG}(\mathrm{P}=0.015)$ expression levels changed significantly (Table II).

Age variation and $C C$. Age is a significant risk factor for tumorigenesis (14). The results of the present study indicated that body homeostasis was altered with age. In normal control women, CA-125, CA-199, ALP, cholesterol and TG indices were significantly different $(\mathrm{P}<0.05)$ between the $<40$ years old age group and the $>50$ years old age group (Table III).

To study the early diagnostic factors that are independent of age, the present study aimed to identify serum indices using two criteria: There was no statistical difference in normal control patients $(\mathrm{P}>0.05)$; and there was a statistical difference in patients with $\mathrm{CC}(\mathrm{P}<0.05$; Table IV; Fig. 2). Comparing the $<40$ years old age group with the $40-50$ years old age group ALP did not change significantly in normal women, but increased significantly in patients with SCC. Taken together, the present data demonstrated that ALP may be a potential predictive factor for the occurrence and progression of $\mathrm{CC}$, particularly in women with SCC among the 40-50 years old age group.

FIGO staging and $C C$. To investigate the association between FIGO staging and $\mathrm{CC}$, the present study further analyzed serological CA-125, CA-199, AFP, ALP, cholesterol and TG levels in patients with SCC, and measured ALP, cholesterol and TG in patients with $\mathrm{AC}$.

For patients with SCC (Table V), only ALP and TG levels increased significantly from Stage I to Stage III/IV CC compared with the normal controls. Furthermore, only ALP and cholesterol levels significantly increased in patients with FIGO Stage I AC compared with the normal controls (Table VI).

Expression pattern of $A L P$ in $C C$. Using the re-analysis strategy, the present study demonstrated that ALP was a potential factor, which may predict the tumorigenesis of CC. Despite the ALP expression levels not exceeding the upper limits of the normal recommended level, the present results indicated that the ALP expression level increased in patients with SCC $(\mathrm{P}<0.001)$ and AC $(\mathrm{P}<0.05)$ compared with the normal controls (Fig. 3A). As shown in Fig. 3B, the ALP expression level was significantly increased between the normal controls and patients with SCC in the 40-50 years old group $(\mathrm{P}<0.001)$ and the $>50$ years old group $(\mathrm{P}<0.01)$. Although the expression levels of ALP also increased with age between the normal controls and patients with $\mathrm{AC}$, the difference was not statistically significant.

As demonstrated by the FIGO staging analysis, the expression levels of ALP were significantly increased in patients with SCC of different FIGO stages, when compared with the normal controls (all $\mathrm{P}<0.001$; Fig. $3 \mathrm{C}$ ). At present, 
Table III. Comparison of seven serum indices by age variation in normal controls.

\begin{tabular}{|c|c|c|c|c|c|c|}
\hline \multirow[b]{3}{*}{ Serum index } & \multicolumn{4}{|c|}{ Age } & & \\
\hline & \multicolumn{2}{|c|}{$<40$ years } & \multicolumn{2}{|c|}{$>50$ years } & \multicolumn{2}{|c|}{$<40$ years vs. $>50$ years } \\
\hline & $\mathrm{n}$ & Mean \pm SD & $\mathrm{n}$ & Mean \pm SD & OR $(95 \% \mathrm{CI})$ & P-value \\
\hline CA-125 & 163 & $17.189 \pm 8.718$ & 27 & $11.888 \pm 5.823$ & $0.868(0.794-0.950)$ & $0.003^{\mathrm{b}}$ \\
\hline CA-199 & 150 & $10.528 \pm 7.794$ & 21 & $14.414 \pm 9.310$ & $1.051(1.001-1.104)$ & $0.038^{\mathrm{a}}$ \\
\hline AFP & 257 & $3.240 \pm 2.151$ & 38 & $3.602 \pm 2.350$ & $1.069(0.932-1.226)$ & 0.339 \\
\hline CEA & 257 & $2.355 \pm 3.005$ & 38 & $2.469 \pm 1.974$ & $1.012(0.910-1.126)$ & 0.821 \\
\hline ALP & 150 & $51.600 \pm 15.236$ & 27 & $67.630 \pm 21.335$ & $1.047(1.023-1.070)$ & $7.57 \times 10^{-4 c}$ \\
\hline Cholesterol & 257 & $4.480 \pm 0.807$ & 37 & $5.203 \pm 0.663$ & $3.143(1.945-5.078)$ & $3.71 \times 10^{-7 \mathrm{c}}$ \\
\hline TG & 257 & $1.015 \pm 0.466$ & 37 & $1.353 \pm 0.410$ & $3.244(1.740-6.045)$ & $3.75 \times 10^{-5 c}$ \\
\hline
\end{tabular}

${ }^{a} \mathrm{P}<0.05,{ }^{\mathrm{b}} \mathrm{P}<0.01,{ }^{\mathrm{C}} \mathrm{P}<0.001$. CA, sugar chain antigen; AFP, $\alpha$ fetoprotein; CEA, carcino-embryonic antigen; ALP, alkaline phosphatase; TG, triglyceride; $\mathrm{SD}$, standard deviation; $\mathrm{OR}$, odds ratio; $\mathrm{CI}$, confidence interval.

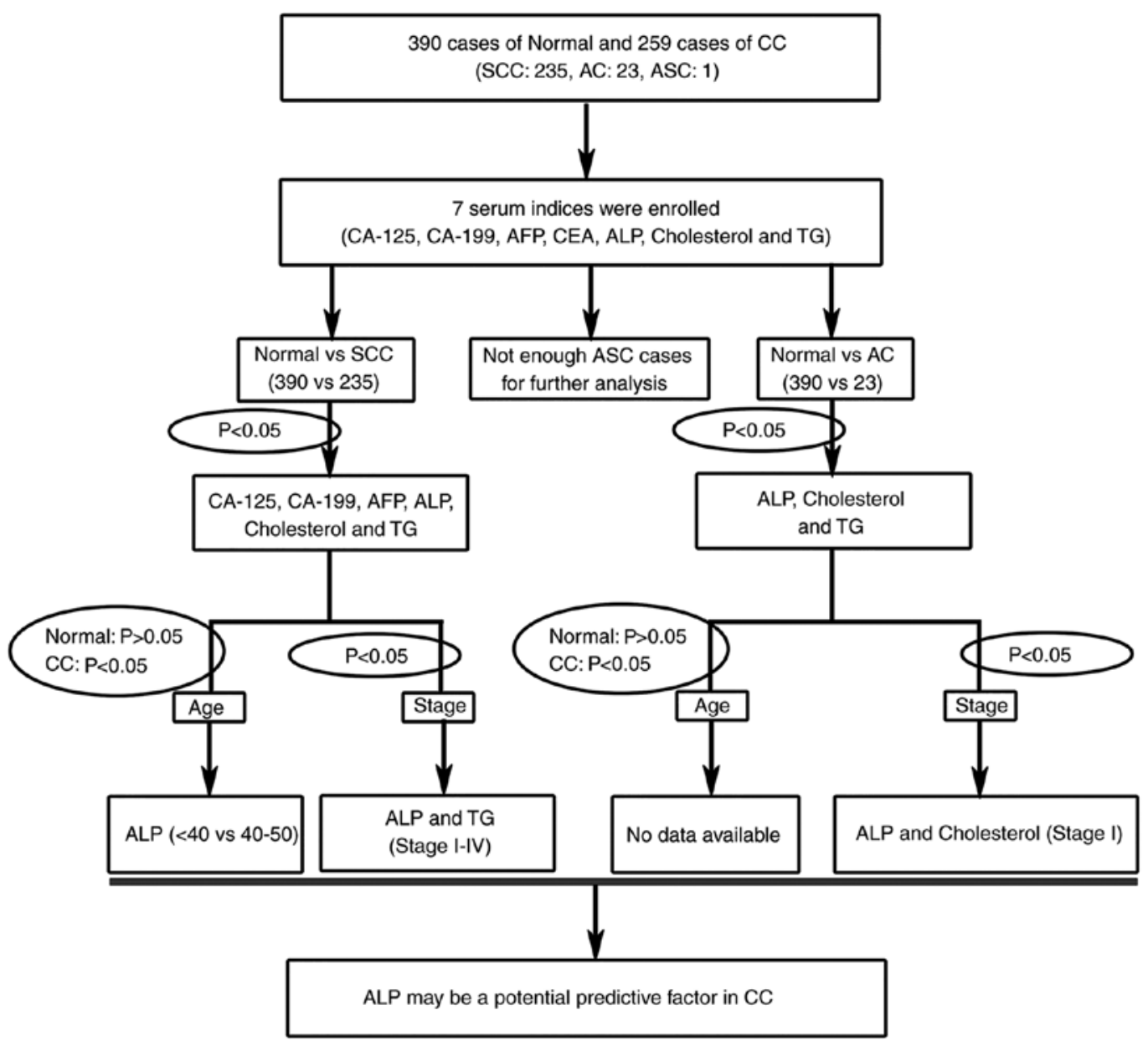

Figure 2. A flow chart of the re-analysis strategy. The re-analysis strategy assessed seven different serum indices. Further verification was based on age variation and The International Federation of Gynecology and Obstetrics staging. CC, cervical cancer; SCC, squamous cell carcinoma; AC, cervical adenocarcinoma; ASC, adenosquamous cervical carcinoma; CA, sugar chain antigen; AFP, $\alpha$ fetoprotein; CEA, carcino-embryonic antigen; ALP, alkaline phosphatase; TG, triglyceride.

the number of $\mathrm{AC}$ cases for comparison across the FIGO stages was limited, and a larger sample size is required to further confirm the significance of ALP and other serum indices. The present study also detected the relative mRNA expression level of the major type of ALP isozyme, TNALP, between the normal control and CC tissues. The RT-qPCR results indicated that TNALP mRNA expression levels were significantly increased in CC tissues compared with normal cervical tissues (Fig. 3D; $\mathrm{P}<0.01$ ). In summary, the results demonstrated that ALP might be a reliable early predictive 
Table IV. Comparison of the seven serum indices by age variation in normal controls, patients with SCC and patients with AC.

A, Normal

\begin{tabular}{|c|c|c|c|c|c|c|}
\hline \multirow[b]{3}{*}{ Serum index } & \multicolumn{4}{|c|}{ Age } & & \\
\hline & \multicolumn{2}{|c|}{$<40$ years } & \multicolumn{2}{|c|}{$40-50$ years } & \multicolumn{2}{|c|}{$<40$ years vs. $40-50$ years } \\
\hline & $\mathrm{n}$ & Mean \pm SD & $\mathrm{n}$ & Mean \pm SD & OR $(95 \% \mathrm{CI})$ & P-value \\
\hline CA-125 & 163 & $17.189 \pm 8.718$ & 56 & $16.850 \pm 8.691$ & $0.995(0.960-1.032)$ & 0.802 \\
\hline CA-199 & 150 & $10.528 \pm 7.794$ & 49 & $9.249 \pm 6.476$ & $0.975(0.930-1.023)$ & 0.301 \\
\hline AFP & 257 & $3.240 \pm 2.151$ & 92 & $4.129 \pm 3.180$ & $1.136(1.039-1.243)$ & $0.014^{\mathrm{a}}$ \\
\hline CEA & 257 & $2.355 \pm 3.005$ & 93 & $2.605 \pm 2.529$ & $1.028(0.952-1.109)$ & 0.474 \\
\hline ALP & 150 & $51.600 \pm 15.236$ & 60 & $50.850 \pm 12.791$ & $0.996(0.976-1.018)$ & 0.737 \\
\hline Cholesterol & 257 & $4.480 \pm 0.807$ & 94 & $4.876 \pm 0.776$ & $1.852(1.360-2.523)$ & $4.84 \times 10^{-5 c}$ \\
\hline TG & 257 & $1.015 \pm 0.466$ & 94 & $1.252 \pm 0.650$ & $2.214(1.400-3.502)$ & $0.002^{\mathrm{b}}$ \\
\hline
\end{tabular}

$\mathrm{B}, \mathrm{SCC}$

\begin{tabular}{|c|c|c|c|c|c|c|}
\hline \multirow[b]{3}{*}{ Serum index } & \multicolumn{4}{|c|}{ Age } & & \\
\hline & \multicolumn{2}{|c|}{$<40$ years } & \multicolumn{2}{|c|}{$40-50$ years } & \multicolumn{2}{|c|}{$<40$ years vs. $40-50$ years } \\
\hline & $\mathrm{n}$ & Mean \pm SD & $\mathrm{n}$ & Mean \pm SD & OR $(95 \% \mathrm{CI})$ & P-value \\
\hline CA-125 & 14 & $19.107 \pm 8.060$ & 54 & $21.213 \pm 21.154$ & $1.007(0.971-1.044)$ & 0.717 \\
\hline CA-199 & 11 & $14.271 \pm 11.415$ & 46 & $19.029 \pm 28.154$ & $1.009(0.976-1.043)$ & 0.587 \\
\hline AFP & 12 & $4.603 \pm 3.513$ & 53 & $2.722 \pm 1.187$ & $0.660(0.473-0.920)$ & 0.093 \\
\hline CEA & 12 & $4.047 \pm 8.508$ & 54 & $3.991 \pm 6.923$ & $0.999(0.915-1.090)$ & 0.981 \\
\hline ALP & 28 & $56.843 \pm 13.926$ & 97 & $64.723 \pm 18.471$ & $1.028(1.001-1.056)$ & $0.039^{\mathrm{a}}$ \\
\hline Cholesterol & 18 & $4.508 \pm 0.691$ & 67 & $4.563 \pm 1.015$ & $1.065(0.610-1.858)$ & 0.828 \\
\hline $\mathrm{TG}$ & 18 & $1.273 \pm 0.678$ & 67 & $1.395 \pm 0.713$ & $1.313(0.579-2.974)$ & 0.518 \\
\hline
\end{tabular}

$\mathrm{C}, \mathrm{AC}$

\begin{tabular}{|c|c|c|c|c|c|c|}
\hline \multirow[b]{3}{*}{ Serum index } & \multicolumn{4}{|c|}{ Age } & & \\
\hline & \multicolumn{2}{|c|}{$<40$ years } & \multicolumn{2}{|c|}{$40-50$ years } & \multicolumn{2}{|c|}{$<40$ years vs. $40-50$ years } \\
\hline & $\mathrm{n}$ & Mean \pm SD & $\mathrm{n}$ & Mean \pm SD & OR $(95 \% \mathrm{CI})$ & P-value \\
\hline CA-125 & 4 & $17.625 \pm 10.356$ & 4 & $37.000 \pm 35.849$ & $1.040(0.962-1.124)$ & 0.365 \\
\hline CA-199 & 4 & $14.740 \pm 8.105$ & 3 & $12.550 \pm 6.856$ & $0.950(0.753-1.198)$ & 0.722 \\
\hline AFP & 4 & $2.115 \pm 1.051$ & 3 & $4.023 \pm 1.932$ & $4.236(0.351-51.105)$ & 0.149 \\
\hline CEA & 4 & $5.020 \pm 6.482$ & 4 & $2.880 \pm 2.929$ & $0.890(0.624-1.269)$ & 0.569 \\
\hline ALP & 5 & $58.180 \pm 7.811$ & 6 & $66.167 \pm 41.732$ & $1.011(0.965-1.058)$ & 0.686 \\
\hline Cholesterol & 4 & $4.878 \pm 0.970$ & 5 & $4.850 \pm 0.768$ & $0.953(0.168-5.395)$ & 0.963 \\
\hline TG & 4 & $1.360 \pm 0.920$ & 5 & $1.604 \pm 0.544$ & $1.808(0.218-15.012)$ & 0.633 \\
\hline
\end{tabular}

${ }^{\mathrm{a}} \mathrm{P}<0.05,{ }^{\mathrm{b}} \mathrm{P}<0.01,{ }^{\mathrm{c}} \mathrm{P}<0.001$. SCC, squamous cell carcinoma; AC, cervical adenocarcinoma; $\mathrm{CA}$, sugar chain antigen; AFP, $\alpha$ fetoprotein; CEA, carcino-embryonic antigen; ALP, alkaline phosphatase; TG, triglyceride; SD, standard deviation; OR, odds ratio; CI, confidence interval.

factor for detecting the development of CC, particularly in 40-50 year-old women.

Adjusted assessment of the serum indices for CC. Liver function is an important factor associated with progression of numerous diseases and tumorigenesis (15). A recent study demonstrated that serological ALP and AST expression levels were significantly increased in patients with gastric cancer (16). Similarly, the present study confirmed that ALP expression levels were also increased in patients with CC. To exclude the effects of liver dysfunction on ALP expression, the present study also assessed seven serum indices between normal control women and patients with $\mathrm{CC}$ using adjusted AST and ALT factors (excluding cases exceeding the normal upper limit of AST and ALT). The present data indicated that the seven serum indices between the normal control women and patients with $\mathrm{CC}$ exhibited similar results in the adjusted assessment (Table VII). 

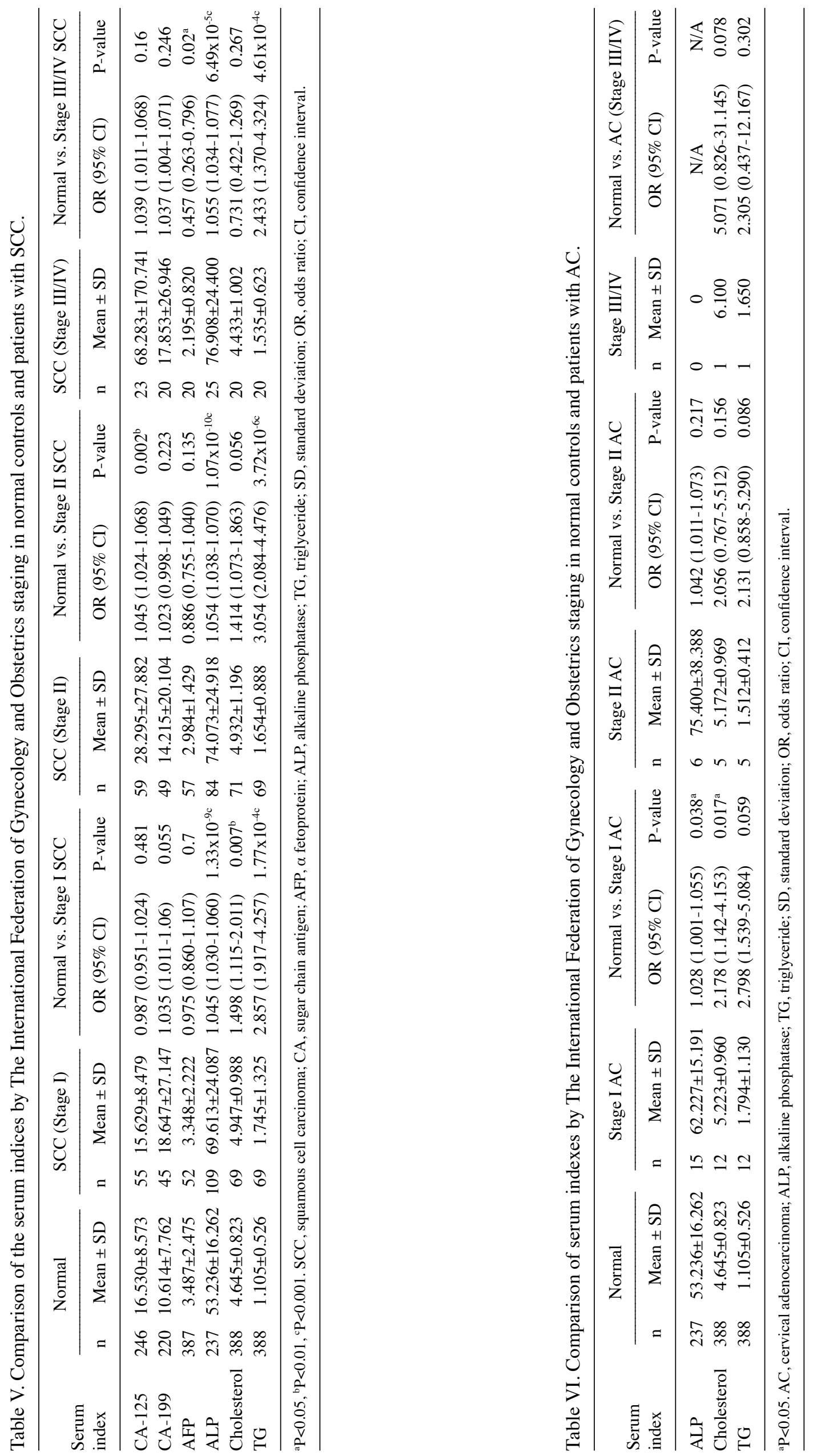
Table VII. Adjusted assessment of the serum indices by aspartate aminotransferase and alanine transferase in normal controls and patients with cervical cancer.

\begin{tabular}{|c|c|c|c|c|c|c|c|c|c|c|}
\hline \multirow[b]{2}{*}{ Serum index } & \multicolumn{2}{|r|}{ Normal } & \multicolumn{2}{|r|}{$\mathrm{SCC}$} & \multicolumn{2}{|c|}{ Normal vs. SCC } & \multicolumn{2}{|r|}{$\mathrm{AC}$} & \multicolumn{2}{|c|}{ Normal vs. AC } \\
\hline & $\mathrm{n}$ & Mean \pm SD & $\mathrm{n}$ & Mean \pm SD & OR $(95 \% \mathrm{CI})$ & P-value & $\mathrm{n}$ & Mean \pm SD & OR $(95 \% \mathrm{CI})$ & P-value \\
\hline CA-125 & 238 & $16.692 \pm 8.634$ & 118 & $29.815 \pm 77.010$ & $1.028(1.011-1.046)$ & 0.067 & 13 & $19.592 \pm 26.488$ & $1.020(0.981-1.060)$ & 0.701 \\
\hline CA-199 & 214 & $10.613 \pm 7.723$ & 99 & $15.477 \pm 21.506$ & $1.026(1.006-1.046)$ & $0.031^{\mathrm{a}}$ & 10 & $10.642 \pm 7.048$ & $1.000(0.921-1.087)$ & 0.991 \\
\hline AFP & 374 & $3.510 \pm 2.502$ & 113 & $2.904 \pm 1.645$ & $0.862(0.761-0.976)$ & $0.003^{\mathrm{b}}$ & 13 & $3.075 \pm 2.198$ & $0.912(0.679-1.224)$ & 0.537 \\
\hline CEA & 375 & $2.431 \pm 2.812$ & 117 & $11.202 \pm 71.674$ & $1.099(1.040-1.161)$ & 0.188 & 13 & $3.703 \pm 3.827$ & $1.074(0.972-1.186)$ & 0.114 \\
\hline ALP & 229 & $52.856 \pm 16.006$ & 200 & $71.745 \pm 23.407$ & $1.055(1.041-1.068)$ & $1.503 \times 10^{-19 c}$ & 17 & $62.459 \pm 13.607$ & $1.030(1.005-1.057)$ & $0.017^{\mathrm{a}}$ \\
\hline Cholesterol & 375 & $4.639 \pm 0.819$ & 148 & $4.839 \pm 1.024$ & $1.289(1.040-1.598)$ & $0.035^{\mathrm{a}}$ & 14 & $5.306 \pm 0.958$ & $2.436(1.333-4.452)$ & $0.003^{\mathrm{b}}$ \\
\hline TG & 375 & $1.100 \pm 0.523$ & 146 & $1.655 \pm 1.081$ & $3.160(2.250-4.440)$ & $1.535 \times 10^{-8 c}$ & 14 & $1.540 \pm 0.709$ & $2.384(1.256-4.525)$ & $0.038^{\mathrm{a}}$ \\
\hline
\end{tabular}

${ }^{a} \mathrm{P}<0.05,{ }^{b} \mathrm{P}<0.01,{ }^{\mathrm{c}} \mathrm{P}<0.001$. SCC, squamous cell carcinoma; AC, cervical adenocarcinoma; $\mathrm{CA}$, sugar chain antigen; AFP, $\alpha$ fetoprotein; CEA, carcino-embryonic antigen; ALP, alkaline phosphatase; TG, triglyceride; SD, standard deviation; OR, odds ratio; CI, confidence interval.

A

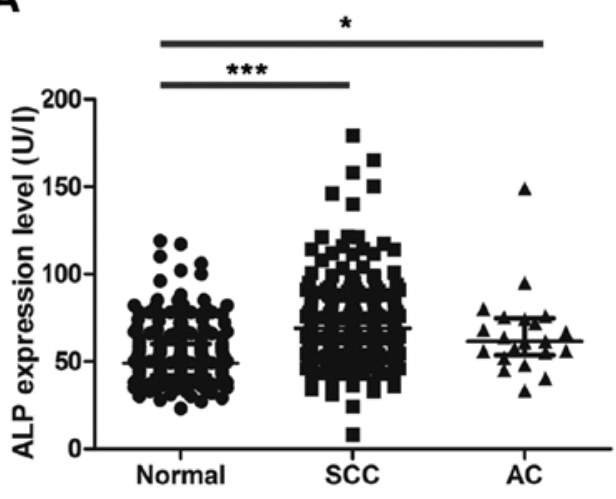

C

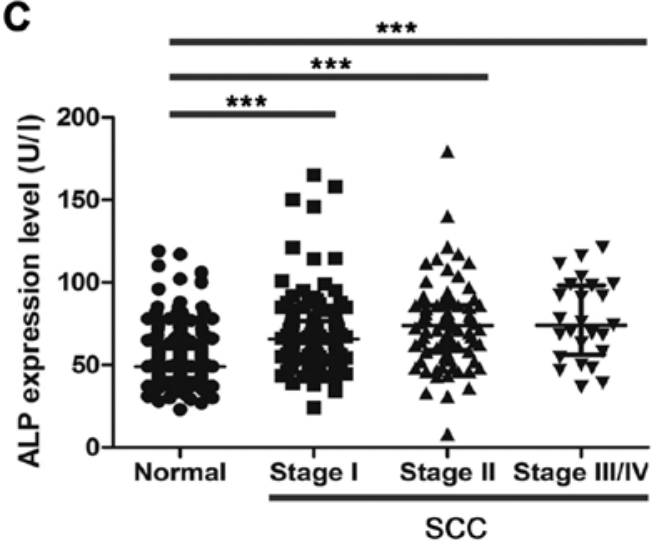

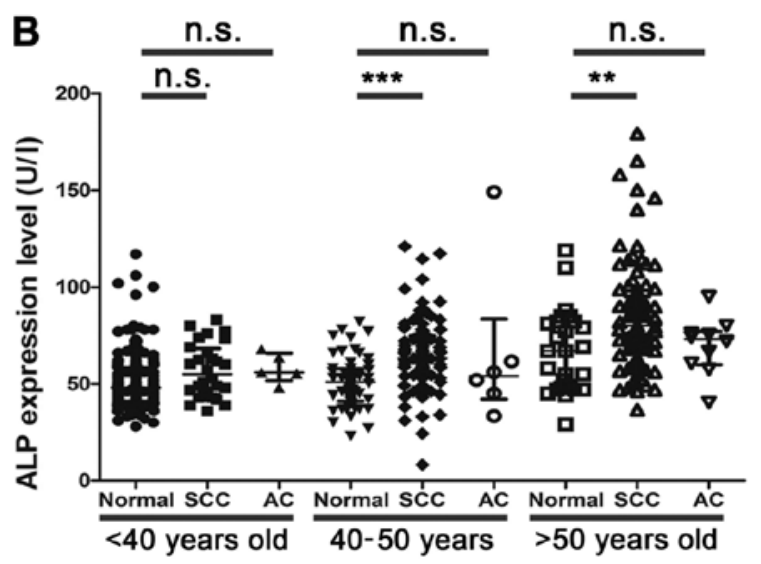

D

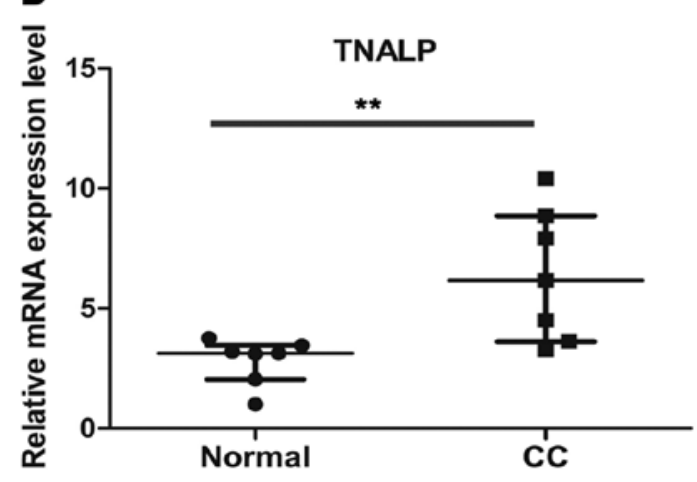

Figure 3. ALP expression level is significantly increased in patients with CC. (A) ALP expression level in normal controls, and patients with SCC and AC by biochemical index detection. (B) Analysis of ALP expression level by age bracket. (C) Analysis of ALP expression level by The International Federation of Gynecology and Obstetrics staging in SCC. (D) Relative mRNA expression level of TNALP in normal cervical tissues and CC tissues. Data are presented as the median and the interquartile range. ${ }^{*} \mathrm{P}<0.05,{ }^{* *} \mathrm{P}<0.01,{ }^{* * *} \mathrm{P}<0.001$. n.s., not significant; ALP, alkaline phosphatase; CC, cervical cancer; $\mathrm{SCC}$, squamous cell carcinoma; AC, cervical adenocarcinoma; TNALP, tissue non-specific isozyme ALP.

\section{Discussion}

In the present study, to determine novel clinical predictive factors for the development of CC, a multistage re-analysis strategy of seven serum indices was systematically conducted. The expression levels of CA-125, CA-199, ALP, cholesterol and TG significantly increased in patients with SCC. In addition, the expression levels of AFP were significantly decreased in patients with SCC. In patients with AC, ALP, cholesterol and TG expression levels significantly increased compared with the normal controls. According to age variation (normal, $\mathrm{P}>0.05 ; \mathrm{CC}, \mathrm{P}<0.05)$ and FIGO staging $(\mathrm{P}<0.05)$, the combined analysis results demonstrated that ALP appears to serve a role in CC. ALP expression significantly increased in patients with SCC and AC compared with the normal controls, indicating its potential role in the diagnosis of $\mathrm{CC}$. 
ALP is a member of the superfamily of metalloenzymes, which catalyze the hydrolysis of phosphoric acid. In humans, there are four types of ALP isozyme, including TNALP, which is primarily expressed in bone tissue, the liver and kidneys, intestinal-type ALP, placental-type ALP and germ cell ALP (17). TNALPs in the bone tissue and liver account for $>90 \%$ of serum ALP and are the most abundantly expressed isoforms in the serum (18). Previous studies have indicated that ALP is a prognostic factor in predicting overall survival in advanced prostate cancer $(19,20)$. However, the role and mechanism of ALP in tumor pathogenesis remain unclear. A previous study demonstrated that ALP functioned as an ectoenzyme in human osteosarcoma cells (21). Another previous study showed that the tumor-derived alkaline phosphatase, biomineralization associated (ALPL) gene, encoding the TNALP protein, was associated with disease-free survival in metastatic prostate cancer and that ALPL promoted the pathogenesis of prostate cancer (22). Osteoblasts abundantly express ALP, which can hydrolyze inorganic pyrophosphate and thus, affect bone mineralization, indicating its roles in osteoblastic activity (23).

Recently, evidence indicated that TNALP could regulate cranial base growth and synchondrosis maturation via mitogen-activated protein kinase (MAPK) signalling (24). Previous studies have also indicated the role of MAPK signaling in tumorigenesis (25-28), and demonstrated that oncogenes promote tumor growth and metastasis through the MAPK signaling pathway (29). Ectopic activation of the MAPK signaling pathway enhances the progression of cancer (30). Furthermore, epidermal growth factor receptor regulated matrix metalloproteinase 1 expression via the MAPK/extracellular signal-regulated kinase signaling pathway in SiHa cells, which has been shown to serve a role in tumor invasion, metastasis and angiogenesis (31).

$\mathrm{CC}$ is a disease caused by multiple pathogenic factors, including age, human papillomavirus (HPV) infection, FIGO stage and menopausal status (32). The present study provided a multiple-stage screening strategy for exploring predictive factors by systematic re-analysis of serum indices, and two key pathogenic factors were selected for the second round of screening. ALP was identified as a potential predictor for CC progression. Combined analysis of ALP with other factors, including HPV infection and cytological examination may optimize the diagnosis of CC. Furthermore, serum ALP levels are determined as part of a routine examination in hospitals and the present study suggested that even routine tests can reveal the occurrence of $\mathrm{CC}$, providing important information for early diagnosis of CC. However, more accurate data acquisition is required to confirm the results of the present study and further elucidate the mechanism underlying the development of CC. Additionally, estradiol was determined to be an important factor for affecting ALP levels between normal women and CC in pre-menopausal women (data not shown), and thus, the correlation between estradiol and ALP levels in normal controls and patients with $\mathrm{CC}$ requires further analysis in future studies.

The present study identified elevated expression levels of ALP in patients with SCC and AC compared with the normal controls. High levels of serum ALP may influence tumorigenesis and may be an important predictive factor in CC. Further studies may focus on the role and mechanism of increased expression of TNALP in CC. The present study provided novel insight into determining an association between ALP and CC.

\section{Acknowledgements}

Not applicable.

\section{Funding}

The present study was supported by The National Natural Science Foundation of China (grant nos. 31701298 and 81402100), The Natural Science Foundation of Jiangsu Province (grant no. BK20170562), The Key Research Foundation of Zhenjiang Social Development (grant nos. SH2017013, SH2017020, SH2016028, SH2016031 and SH2014026), The Key Research Foundation of Zhenjiang Health Science and Technology (grant no. SHW2016001), The Science Foundation of Doctorate Research of Affiliated Hospital of Jiangsu University (grant no. jdfyRC2016005), Suzhou Key Medical Center (grant no. SZZX201505), Suzhou Introduced Project of Clinical Medical Expert Team (grant no. SZYJTD201708), Jiangsu Provincial Medical Innovation Team (grant no. CXTDB2017013), The Foundation of Health and Family Planning Commission of Jiangsu Province (grant no. Q201408), The Foundation for Young Medical Talents of Jiangsu province (grant no. QNRC2016840) and The Six Talent Peaks Project in Jiangsu Province (grant no. WSW-007).

\section{Availability of data and materials}

The datasets used and/or analyzed during the present study are available from the corresponding author on reasonable request.

\section{Authors' contributions}

$\mathrm{JY}, \mathrm{JF}$ and $\mathrm{XH}$ contributed to the conception and design of the study. QZ, BZ, XC, CS, YZ, XL, WC, MW and YY contributed to the acquisition, analysis and interpretation of data. XD, HL and CQ performed the morphological experiments. BX, JL, $\mathrm{BC}$ and $\mathrm{PY}$ were involved in sample collection and performing molecular experiments.

\section{Ethics approval and consent to participate}

The present study was approved by The Ethics Committee for Biomedical Research at Affiliated Hospital of Jiangsu University (approval no. 20120019), and conducted according to the guidelines for clinical retrospective studies. All participants were fully informed of the purpose and significance of the study. Written informed consent was obtained from all the participants for participation.

\section{Patient consent for publication}

All patients provided consent for the use of their samples in scientific research.

\section{Competing interests}

The authors declare that they have no competing interests. 


\section{References}

1. Jemal A, Bray F, Center MM, Ferlay J, Ward E and Forman D Global cancer statistics. CA Cancer J Clin 61: 69-90, 2011.

2. Chen W, Zheng R, Baade PD, Zhang S, Zeng H, Bray F, Jemal A, Yu XQ and He J: Cancer statistics in China, 2015. CA Cancer J Clin 66: 115-132, 2016.

3. Chen P, Jiao L and Wang DB: Squamous cell carcinoma antigen expression in tumor cells is associated with the chemosensitivity and survival of patients with cervical cancer receiving docetaxel-carboplatin-based neoadjuvant chemotherapy. Oncol Lett 13: 1235-1241, 2017.

4. Molina R, Filella X, Augé JM, Fuentes R, Bover I, Rifa J, Moreno V, Canals E, Viñolas N, Marquez A, et al: Tumor markers (CEA, CA 125, CYFRA 21-1, SCC and NSE) in patients with non-small cell lung cancer as an aid in histological diagnosis and prognosis. Comparison with the main clinical and pathological prognostic factors. Tumour Biol 24: 209-218, 2003

5. Catanzaro JM, Guerriero JL, Liu J, Ullman E, Sheshadri N, Chen JJ and Zong WX: Elevated expression of squamous cell carcinoma antigen (SCCA) is associated with human breast carcinoma. PLoS One 6: e19096, 2011.

6. Sánchez Vega JF, Murillo Bacilio MDR, Vintimilla Condoy AS Palta González AM, Crespo Astudillo JA and Mora-Bravo FG: Predictive equation of metastasis in patients with malignant ovarian epithelial tumors with the $\mathrm{Ca}-125$ marker. BMC Cancer 18: 587, 2018.

7. Malati T: Tumour markers: An overview. Indian J Clin Biochem 22: 17-31, 2007.

8. Lebrecht A, Ludwig E, Huber A, Klein M, Schneeberger C, Tempfer C, Koelbl H and Hefler L: Serum vascular endothelia growth factor and serum leptin in patients with cervical cancer. Gynecol Oncol 85: 32-35, 2002.

9. Fuso L, Mazzola S, Marocco F, Ferrero A, Dompè D, Carus AP and Zola P: Pretreatment serum hemoglobin level as a predictive factor of response to neoadjuvant chemotherapy in patients with locally advanced squamous cervical carcinoma: A preliminary report. Gynecol Oncol 99 (Suppl 1): S187-S191, 2005.

10. Bhatla N, Aoki D, Sharma DN and Sankaranarayanan R: Cancer of the cervix uteri. Int J Gynaecol Obstet 143 (Suppl 2): S22-S36, 2018.

11. US Preventive Services Task Force, Curry SJ, Krist AH, Owens DK, Barry MJ, Caughey AB, Davidson KW, Doubeni CA, Epling JW Jr, Kemper AR, et al: Screening for cervical cancer: US preventive services task force recommendation statement. JAMA 320: 674-686, 2018.

12. Livak KJ and Schmittgen TD: Analysis of relative gene expression data using real-time quantitative PCR and the 2(-Delta Delta C(T)) method. Methods 25: 402-408, 2001.

13. Herfs M, Yamamoto Y, Laury A, Wang X, Nucci MR, McLaughlin-Drubin ME, Münger K, Feldman S, McKeon FD, Xian W and Crum CP: A discrete population of squamocolumnar junction cells implicated in the pathogenesis of cervical cancer. Proc Natl Acad Sci USA 109: 10516-10521, 2012.

14. Quinn BA, Deng X, Colton A, Bandyopadhyay D, Carter JS and Fields EC: Increasing age predicts poor cervical cancer prognosis with subsequent effect on treatment and overall survival. Brachytherapy 18: 29-37, 2019.

15. Zhang C, Wang H, Ning Z, Xu L, Zhuang L, Wang P and Meng Z: Serum liver enzymes serve as prognostic factors in patients with intrahepatic cholangiocarcinoma. Onco Targets Ther 10: 1441-1449, 2017.

16. Fuchs CS, Muro K, Tomasek J, Van Cutsem E, Cho JY, Oh SC, Safran H, Bodoky G, Chau I, Shimada Y, et al: Prognostic factor analysis of overall survival in gastric cancer from two phase III studies of second-line ramucirumab (REGARD and RAINBOW) using pooled patient data. J Gastric Cancer 17: 132-144, 2017.

17. Haarhaus M, Brandenburg V, Kalantar-Zadeh K, Stenvinkel P and Magnusson P: Alkaline phosphatase: A novel treatment target for cardiovascular disease in CKD. Nat Rev Nephrol 13: 429-442, 2017.
18. Magnusson P, Degerblad M, Sääf M, Larsson L and Thorén M: Different responses of bone alkaline phosphatase isoforms during recombinant insulin-like growth factor-i (IGF-I) and during growth hormone therapy in adults with growth hormone deficiency. J Bone Miner Res 12: 210-220, 1997.

19. Metwalli AR, Rosner IL, Cullen J, Chen Y, Brand T, Brassell SA, Lesperance J, Porter C, Sterbis J and McLeod DG: Elevated alkaline phosphatase velocity strongly predicts overall survival and the risk of bone metastases in castrate-resistant prostate cancer. Urol Oncol 32: 761-768, 2014.

20. Sonpavde G, Pond GR, Berry WR, de Wit R, Armstrong AJ, Eisenberger MA and Tannock IF: Serum alkaline phosphatase changes predict survival independent of PSA changes in men with castration-resistant prostate cancer and bone metastasis receiving chemotherapy. Urol Oncol 30: 607-613, 2012.

21. Fedde KN, Lane CC and Whyte MP: Alkaline phosphatase is an ectoenzyme that acts on micromolar concentrations of natural substrates at physiologic $\mathrm{pH}$ in human osteosarcoma (SAOS-2) cells. Arch Biochem Biophys 264: 400-409, 1998.

22. Rao SR, Snaith AE, Marino D, Cheng X, Lwin ST, Orriss IR, Hamdy FC and Edwards CM: Tumour-derived alkaline phosphatase regulates tumour growth, epithelial plasticity and disease-free survival in metastatic prostate cancer. Br J Cancer 116: 227-236, 2017.

23. Millán JL: The role of phosphatases in the initiation of skeletal mineralization. Calcif Tissue Int 93: 299-306, 2013.

24. Nam HK, Sharma M, Liu J and Hatch NE: Tissue nonspecific alkaline phosphatase (TNAP) regulates cranial base growth and synchondrosis maturation. Front Physiol 8: 161, 2017.

25. Shao J, Wang C, Li L, Liang H, Dai J, Ling X and Tang H Luteoloside inhibits proliferation and promotes intrinsic and extrinsic pathway-mediated apoptosis involving MAPK and mTOR signaling pathways in human cervical cancer cells. Int J Mol Sci 19: E1664, 2018.

26. Hua FF, Liu SS, Zhu LH, Wang YH, Liang X, Ma N and Shi HR: MiRNA-338-3p regulates cervical cancer cells proliferation by targeting MACC1 through MAPK signaling pathway. Eur Rev Med Pharmacol Sci 21: 5342-5352, 2017.

27. Sun Q, Liang Y, Zhang T, Wang K and Yang X: ER- $\alpha 36$ mediates estrogen-stimulated MAPK/ERK activation and regulates migration, invasion, proliferation in cervical cancer cells. Biochem Biophys Res Commun 487: 625-632, 2017.

28. Li S, Ma YM, Zheng PS and Zhang P: GDF15 promotes the proliferation of cervical cancer cells by phosphorylating AKT1 and Erk1/2 through the receptor ErbB2. J Exp Clin Cancer Res 37: 80, 2018

29. Yang XL, Liu KY, Lin FJ, Shi HM and Ou ZL: CCL28 promotes breast cancer growth and metastasis through MAPK-mediated cellular anti-apoptosis and pro-metastasis. Oncol Rep 38: 1393-1401, 2017.

30. Wang C, Li P, Xuan J, Zhu C, Liu J, Shan L, Du Q, Ren Y and Ye J: Cholesterol enhances colorectal cancer progression via ROS elevation and MAPK signaling pathway activation. Cell Physiol Biochem 42: 729-742, 2017.

31. Zhang Z, Wang L, Du J, Li Y, Yang H, Li C, Li H and Hu H: Lipid raft localization of epidermal growth factor receptor alters matrix metalloproteinase-1 expression in $\mathrm{SiHa}$ cells via the MAPK/ERK signaling pathway. Oncol Lett 12: 4991-4998, 2016.

32. Cohen PA, Jhingran A, Oaknin A and Denny L: Cervical cancer. Lancet 393: 169-182, 2019.

This work is licensed under a Creative Commons Attribution-NonCommercial-NoDerivatives 4.0 International (CC BY-NC-ND 4.0) License. 